Voix et Images

\title{
Frère de mon âme et de mon art : Claude-Henri Grignon-Alfred DesRochers (1930-1942)
}

\section{Yvette Francoli}

Volume 16, numéro 1 (46), automne 1990

Les correspondants littéraires d'Alfred DesRochers

URI : https://id.erudit.org/iderudit/200872ar

DOI : https://doi.org/10.7202/200872ar

Aller au sommaire du numéro

Éditeur(s)

Université du Québec à Montréal

ISSN

0318-9201 (imprimé)

1705-933X (numérique)

Découvrir la revue

Citer cet article

Francoli, Y. (1990). Frère de mon âme et de mon art : Claude-Henri

Grignon-Alfred DesRochers (1930-1942). Voix et Images, 16(1), 44-52.

https://doi.org/10.7202/200872ar d'utilisation que vous pouvez consulter en ligne.

https://apropos.erudit.org/fr/usagers/politique-dutilisation/ 


\section{Frère de mon âme et de mon art: Claude-Henri Grignon- Alfred DesRochers (1930-1942)}

\section{par Yvette Francoli, Université de Sherbrooke}

La correspondance entre Claude-Henri Grignon et Alfred DesRochers s'échelonne du 12 mars 1930 au 28 mars 1942. En tout, vingt-deux lettres: six en 1930, huit en 1931, sept en 1933 et finalement une en 1942. Elles sont toutes de Grignon à l'exception d'une de DesRochers datée du 16 janvier 1931. Toutes celles du poète ont disparu. Peut-être ont-elle été détruites par Grignon lui-même, vers la fin de sa vie, au cours du grand élagage qu'il effectua dans ses papiers personnels, certains jours où il ne dominait plus sa maladie.

Nul ne sait pourquoi ils ont subitement cessé de correspondre. On s'étonne toutefois, connaissant l'adoration que Grignon vouait au poète de l'Estrie, que cette amitié ait tourné court. Étonnant aussi le fait que DesRochers qui, selon l'habitude de l'époque, gardait une copie carbone de ses lettres, n'ait retenu de cet échange épistolaire que celle datée du 16 janvier 1931. Était-ce à ses yeux la seule digne d'être conservée? Il y confiait en effet un projet qui lui tenait à cour (mais combien irréalisable à cette époque): fonder une revue exclusivement canadienne qui ne publierait que des œuvres de valeur, pour lesquelles il se disait prêt à payer des sommes très élevées, seule garantie pour obtenir des textes bien travaillés et relus. En l'absence des lettres de DesRochers, il ne nous a pas été facile de reconstituer sa pensée. Grignon, par chance, a la plume prolixe et l'on devine souvent à ses réponses les sujets d'intérêt et les préoccupations des deux amis.

C'est Grignon qui avait initié cette correspondance pour témoigner au poète d'À l'ombre de l'Orford toute l'admiration étonnante qu'avaient soulevée dans [s]on âme [ses] vers rudes, mâles, énergiques, si sombres de mélancolie parfois et si grands de pensée humaine. Il avouait le faire avec toute la fougue et tout l'amour dont [il était] capable [lorsqu'il] découvr[ait] la Sainte Poésie dans un cœur d'homme (12 mai 1930). Grignon, on le sait, aimait ou détestait avec passion ou véhémence. Envers DesRochers, il s'agit de vénération: Mon cher Dieu, va-t-il jusqu'à lui écrire, ou encore, Frère de mon âme et de mon 
art. S'ils sont nombreux, les écrivains et critiques qu'il a férocement empalés - selon son expresion favorite - et le plus souvent fort injustement, rares sont ceux qui peuvent se flatter d'avoir déclenché chez lui une telle émotion, à part peut-être son idole Léon Bloy. DesRochers vous arrache des larmes, écrira-t-il, en 1936, dans ses Pamphlets. Il est, selon lui, le modèle poétique par excellence, ainsi qu'il le lui écrit dès ses premières lettres: 'Pas un poète canadien n'a saisi et compris et exprimé avant vous l'âpre vie des bois et des fermes, campé des hommes vrais aux testicules naturels. (12 décembre 1930) C'est par cet assaut de virilité dans la digne lignée des coureurs de bois que Grignon, fervent défenseur de l'art régionaliste, lui signifiait son horreur de la poésie anémique et des jolis vers parnassiens des exotistes et du gros Paul (Morin) en particulier. Mais DesRochers n'était pas prêt à se cantonner dans le style terroiriste comme l'eût voulu Grignon, et c'est ce qui, à notre avis, devait graduellement les éloigner l'un de l'autre.

Quand Grignon apprend par exemple, lors d'une réunion chez Pelletier, que DesRochers préfere l'Offrande aux vierges folles à À l'ombre de l'Orford, il ne peut taire sa déception. Certes, il avait accueilli à genoux [...] ces admirables vierges qui lui donnaient toutes sortes de frissons (26 décembre 1930), mais il ne peut admettre que DesRochers considère l'Orford lettre morte :

Si c'est vrai, mon cher Fred, tu es inconscient ou tu veux te payer la tête de quelqu'un. Ça commence à devenir plus grave. Je considère À l'ombre [...] bien supérieur à l'autre. Par la pensée d'abord, plus forte, plus claire, plus humaine et plus utile; et ensuite par le style, plus précis, plus sonore et beaucoup plus poétique à mon humble point dé vue. Tu considérerais donc ta Vierge folle [...] comme l'enfant prodige ou l'enfant de l'amour. Laisse-moi te dire qu'il y a des fils naturels qui sont loin d'être fortement constitués ou moralement intéressants, tandis que certains enfants légitimes ne manquent pas d'étonner l'univers. Je veux là-dessus avoir ton opinion nette et claire. Je t'avoue que ce soir-là Choquette partageait mon idée en masse. Mais un poète est mauvais juge. Pelletier a penché un peu vers toi. (26 décembre 1930).

DesRochers, dans la seule lettre que nous possédons, lui confirme en effet que la veine de l'Orford ne l'intéresse plus:

Pardonne-moi de t'écrire aussi sèchement en réponse à d'aussi enthousiastes versets. C'est une idée fixe chez moi. Je descends d'une lignée de colons. L'Engagé de Maria Chapdelaine, c'est mon père, mon frère, mes grands-pères. Christ de Viarge, faut qu'je l'arrache, c'te souche-là ! Et tant que la souche n'est pas arrachée, tout peut aller en décadence, c'est la souche qui compte! 
Il y a des familles qui sont comme ça: il faut qu'elles fassent de la terre neuve. Aussitôt qu'une terre est défrichée, qu'on pourrait jouir de ses récoltes, on l'abandonne pour aller en ouvrir une autre. C'est mon cas, comme ce fut le cas de tous les miens. La veine de l'Orford ne m'intéresse plus. Je ne pourrais rien faire de bien si je continuais à l'exploiter; je tomberais dans le terroirisme de Blanche Lamontagne. J'achève un roman lyrique, composé de petits poèmes superposés, sans récitatif. Toute l'action repose dans la gradation. Une fois fini, l'amour, comme thème littéraire, ne me dira plus rien. Il me faudra du nouveau, des souches! (16 janvier 1931)

Grignon ne se laissera pas affecter par cette divergence d'opinion. Il croit au contraire avoir rencontré en DesRochers son frère jumeau. Le poète de l'Orford est, comme lui, un autodidacte qui n'a jamais fait partie d'aucune école, qui n'a jamais respiré l'air malsain des cénacles: Je suis, lui écrira-t-il, le 15 janvier 1931, le seul écrivain de chez nous débarrassé de toutes les entraves d'écoles ou de langues. J'ai jeté au ruisseau cette pénible défroque qu'on appelle la méthode analytique [...]. Comme lui, également, DesRochers a le grand mérite d'utiliser une langue sauvage et si humaine: la langue canadienne, ce qui, ainsi qu'il le soulignera dans ses Pamphlets, n'est pas le cas d'un Morin, d'un Nelligan, d'un Chopin, d'un Lozeau qui écrivent le français de France et qui sont incapables de l'écrire comme les Français eux-mêmes:

Toi, au moins, tu te déboutonnes et tu marches à pas carrés dans la m... ou la terre des ancêtres et c'est pourquoi je place tes poèmes aux côtés de Choquette entre Flaubert et Baudelaire [...]. J'ai toujours prophétisé que notre vraie littérature sortirait des bois et de la terre, jamais des bibliothèques et des villes. (26 décembre 1930)

C'est pour le prouver que deux ans plus tard, il publiera Un homme et son péché, considéré comme notre meilleur roman de mœurs paysannes ${ }^{1}$. Ce que Grignon apprécie également dans la poésie de DesRochers, c'est l'absence des clichés et des poncifs habituels:

Je t'embrasse heureux lyrique canadien qui passera à la postérité sans avoir une seule fois parlé du "majestueux SaintLaurent" et des "blés d'or». Sois béni éternellement au royaume du langage rustique, mâle, sapineux et bourrasque, d'où sont bannis tous les castrés et tous les collets hauts d'une littérature savonneuse pour vieilles couturières en chaleur.

Enfin, Grignon qui, à cette époque, se trouvait dans une situation extrêmement difficile, sans argent, presque sans amis (28 septembre 1930), éprouvait sans doute le besoin de sortir de son isolement, de sa

1 Jean-Charles Harvey, "Sacré Valdombre, va!», le Jour, 10 septembre 1938. 
détresse morale et, dans un geste désespéré, tendait la main au poète dont il connaissait la générosité de cœur ${ }^{2}$. DesRochers répondra aussitôt à cet appel à l'aide par des lettres qui lui font $d u$ beau dans l'âme (28 juin 1930) et qui lui apportent toujours un regain de vigueur et de santé (16 janvier 1931).

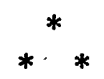

Dès sa deuxième lettre, Grignon, s'enhardissant, réclame de la part du poète un sonnet ou une pièce pas trop longue de [sa] caractéristique plume pour publication dans le Cri du Nord, cette feuille solennelle et artistique qu'il a l'intention de lancer: Faites cela par amour de l'art et non pas par amour de moi! Ce ton passionné laisserait entendre que DesRochers a répondu à ses lettres avec la même fougue. Et, au nom de cette amtié naissante, Grignon, l'irréductible, est prêt à toutes les concessions. C'est tête basse, toute honte bue, qu'il accepte humblement d'avoir eu le tort de critiquer injustement Emile Coderre (ami intime de DesRochers):

Ce que vous dites de Coderre est vrai. Je me repends aujour'd'hui d'avoir été aussi cruel. Mais j'étais jeune et influencé par les jaloux. Ce ne sera plus. Ses dernières pièces sont fort originales (j'en ai lues). Dites bien à Coderre que son dernier genre m'enthousiasme. Il aura comme vous, comme Choquette, comme Éva Senécal, la première page!

DesRochers, qui ne lui en veut pas, lui répond par une invitation chez le mécène Florian Fortin, en l'honneur du passage à Sherbrooke de Louis Dantin, le 30 août 1930 . Grignon se propose d'y lire une "Apologie de la Course dans l'aurore" d'Éva Senécal de sa voix de prophète manqué (il ne la terminera jamais). Il doit se rendre à cette soirée en roaster avec Pierre Dansereau. Malheureusement, ce dernier a un empêchement et Grignon, qui est dans la misère, ne pourra se payer le voyage à Sherbrooke: Tous mes projets s'effacent comme nuages à l'horizon. Il est désespéré et se lamente en des termes fort pathétiques: Je vous souhaite de ne jamais connaître un pareil état qui vous arrache des larmes et des rugissements de lion en captivité. Le sort ne m'aura pas épargné! (11 septembre 1930)

Et soudain, au détour d'une phrase, c'est le tutoiement. Il juge sans doute que sa grande détresse et ses confidences l'y autorisent.

2 DesRochers avait déjà une certaine influence. C'est chez lui qu'avait coutume de se rencontrer le groupe Robert Choquette, Émile Coderre, Alphonse Désilets, Bruchési, Éva Senécal. Grignon comptait peut-être sur cette amitié pour sortir de l'ombre, de cette conspiration du silence où, disait-il, le tenait la critique littéraire canadienne-française. 
Avec ce nouveau témoignage d'amitié renaît l'espoir: N'importe je triompherai un jour!, mots grandioses qui retentissent dans la nuit comme le célèbre Paris, à nous deux maintenant, de Rastignac. Mais avant le triomphe, d'autres déboires et déceptions l'attendent... à commencer par son Cri du Nord qui, de son propre avis, n'aura été qu'un hoquet vulgaire. Il tient responsables de ce nouvel échec un certain ministre de la couronne québecquoise et le soi-disant milieu intellectuel de cette province avachie du genre [Édouard] Montpetit et les ruffians de la politique comme Athanase [David]. Parmi ses ennemis, il y a aussi l'éditeur Édouard Garand et quelques autres salauds des lettres commercialisées qui lui offrent un salaire dérisoire en échange de ses articles de critique ou qui simplement les refusent. Il y a surtout les pontifs de la critique et parmi eux, Louis Dantin qui a le don de réveiller en [lui] un vieux fonds de méchanceté normande.

L'année 1930 touche à sa fin. Grignon continue à parler de ses états d'âme et de sa misère matérielle. Il passe la nuit de Noël à écrire une épître de quatre longues pages à DesRochers parce que cela [lui] fait du.bien et [le] repose, mais il le fait surtout en signe d'amitié, amitié passionnée si l'on en juge par le ton de ses paroles:

Je veux bien t'accorder une heure parce que je t'aime absolument, parce que j'ai pour ton talent une admiration sincère qui peut aller jusqu'à la folie. Il y a de l'architecture dans tes vers classiques et de la grandeur dans ta pensée... (26 décembre 1930)

Il se propose d'écrire un article d'au moins vingt-cinq pages sur le "Prophète de l'Orford", mais il n'y parvient pas: Quatre fois j'ai commencé mon article, quatre fois je l'ai détruit le trouvant plat, incohérent, mal bâti. Il ne l'écrira jamais. Pour le moment, le fait qu'il crève de faim est en grande partie responsable de cette navrante stérilité: Lorsqu'on souffre réellement, on n'écrit pas... Je voudrais posséder les revenus de M. Paul Morin: j'écrirais autre chose que de la cendre ou de l'or artificiel.

À cette époque, Grignon souffre surtout du complexe de persécution. Il se croit la cible unique des critiques de son temps. Or, c'est pour se délivrer des griffes de l'ennemi qui veut sa perte et son scandale qu'il rêve d'écrire ce livre des vérités où il irait avec une allure endiablée, sabrant ici, tirant là, saccageant tout le patrimoine tandis qu'après la bataille surgiraient des débris, les vrais monuments des seuls et rares écrivains dignes de porter ce nom. Ils sont peu nombreux en 1931: quelques poètes, DesRochers, Senécal, Coderre, Choquette, et lui-même, bien sûr. Cet ouvrage qui devait tout d'abord s'intituler le Livre de l'ennemi ou quelques vérités scandaleuses sur la littérature canadienne-française, c'est Ombres et Clameurs dans lequel il se proposait de démontrer que la critique telle que pratiquée au Québec et copiée de la critique 
française ne valait rien et n'était qu'une ordure de plus ajoutée à l'immense tas de fumier. Rancune et rancœur habitent le cœur meurtri de Grignon l'incompris. Nul doute qu'il suit les traces d'Albert Pelletier qui venait de publier Carquois (1931), cette féroce critique du milieu intellectuel et moral de la province dans laquelle Grignon s'était réjoui de ne pas y voir les noms des gros pansus Montpetit, Roy, Chartier, Lozeau (11 février 1931): C'est de la bonne critique, mais je doute qu'elle sera goûtée et comprise de nos soi-disant intellectuels.

Quoi qu'il en soit, Grignon est bien résolu cette année-là à vivre de sa plume. Il propose à DesRochers de mettre en commun leurs efforts pour lancer une revue d'art, indépendante, libre de toute politique et de toute école littéraire:

Pourquoi ne partirions-nous pas ensemble cette feuille? On pourrait $y$ intéresser $M^{\text {lle }}$ Gaudet. Toi, tu es connu dans un certain milieu d'affaires et journalistique. Tu en serais le comptable et le gérant. On pourrait imprimer à Sherbrooke; avoir une liste importante de tous les professionnels de la province et autant que possible marcher par abonnement au lieu d'une vente au comptoir, ce qui ne nous réussirait pas étant donné que notre revue ne ressemblerait nullement à un magazine américain (genre Revue moderne) ou à une Vie parisienne pornographique. Sous une couverture sobre nous étalerions notre nudité et nous pourrions parler librement. Ce serait là un coup formidable porté aux chers abbés, "qu'a mis le roi" [Camille Roy], aux snobs genre Morin, aux taureaux genre Charbonneau, aux chanteurs genre Desaulniers, à tous les exotistes foireux, à tous les régionalistes empêtrés dans la boue artificielle des labeurs et à tous les bas-bleus passés, présents et à venir. (15 janvier 1931)

Ce projet n'aura pas de suite, mais un mois plus tard, Grignon est heureux d'apprendre à son ami qu'il a été nommé critique hebdomadaire au Petit Journal (80 000 lecteurs), ce qui va lui permettre de subsister. Â partir de cette date, ses lettres seront moins longues (une page tout au plus) et moins fréquentes:

J'écris énormément ces temps-ci. Rien de sérieux, excepté mon article sur l'abbé Groulx, paru dans le Canada... C'est là le commencement seulement. Tu vas en voir d'autres constellations dans le Canada avant longtemps. (15 août 1931)

Le moral est temporairement à la hausse. Grignon ne geint plus, il plastronne. Son article sur Groulx est

un maître morceau de prose vigoureuse et de pensée forte. Ça prendrait au taux du change courant 5 Chartier, 5 Camille Roy, 3 Harry Bernard, 3 Maurice Hébert, 3 Jean-Charles Harvey, 


\section{Montpetit et 50 Bruchési pour égaler une telle pièce d'argent} qui n'a cours qu'en pays civilisé. (15 août 1931)

Grignon, qui a subitement repris goût à la vie, laṇce des invitations à Sainte-Adèle, promet de la truite rouge, des lits de plume... À sa grande joie, DesRochers s'empressera d'annoncer son arrivée avec force blasphèmes et rugissements, escorté d'une bande de femelles et d'homosexuels. Tant mieux... J'accepte, répond Grignon enchanté par le langage de plus en plus vert de son ami (22 mai 1931) et, gagné par tant de truculence, il joue à son tour les joyeux lurons, forts en gueule, gros buveurs, gros mangeurs et trousseurs de jupons. Il est question à plusieurs reprises des formes voluptueuses de sa corpulente épouse qui lui procure plus de joie que dix poétesses couchées côte à côte. Ce qui ne saurait surprendre, ces dernières n'étant que de vieilles juments ou de vulgaires bas-bleus et j'en passe..., à l'exception d'Éva Senécal, de Françoise Gaudet dont il compare l'âme à un sous-bois et qui lui semble avoir beaucoup de talent, surtout depuis qu'elle a accepté ses conseils et compris ce qu'il a voulu dire quand il l'avait maltraitée un peu trop durement dans une critique au vitriol (voir lettre du 26 décembre 1930).

Et soudain, c'est le silence, un silence qui durera deux ans, du 25 septembre 1931 au 10 avril 1933. Les lettres de cette période ontelles disparu? S'étaient-ils moins écrit?

Dès septembre 1931, Grignon s'excusait de ne pas répondre d'une façon plus ministérielle aux lettres de son ami:

Il faut me pardonner mon cher paragrapheur et comprendre que je traverse en ce moment le Rubicon de tous les tracas et de toutes les misères imaginables, sans compter celles si nombreuses qu'invente mon imagination diabolique afin de me faire souffrir davantage. Cette vie est un enfer et j'en rends grâce à Dieu en retour de tous les crimes que j'ai commis, et que je pourrais commettre encore étant donné ma maudite nature.

Grignon, acculé à la misère, devait finalement obtenir, grâce à l'intervention de son père spirituel (et à l'occasion père nourrissier!), Olivar Asselin, un poste de quelques mois à titre d'inspecteur des fonds de chômage au ministère de la Colonisation à Québec. Mais cela ne devait régler que temporairement ses soucis d'argent. DesRochers, de son côté, tentera de l'aider comme il pourra, ainsi qu'en témoigne une lettre inédite que lui envoie en retour Olivar Asselin:

J'ai bien noté votre prière d'envoyer cet argent à Valdombre, mais cette démarche serait sûrement au-dessus de mes forces, car il y $a$ en Valdombre un mendiant ingrat qui ne se fait pas scrupule de se doubler d'un hidalgo à l'occasion. En d'autres termes, imaginez vous-même les ruses de trappeur qu'il 
faudra employer pour faire passer les $\mathbf{3} \$$ de votre gousset dans celui de votre ami [...]. (2 juin 1933)

C'est finalement un mandat-poste de $5 \$$ que DesRochers fera parvenir à Valdombre, manne céleste qui lui redonne foi en Dieu et en l'humanité: Si j'ai eu des amis ingrats par le passé, les rares qui me restent aujourd'hui valent de l'or, pour employer une expression précieuse dans le grand monde commercial [...]. (15 juin 1933)

Cette année-là, Grignon présente Ombres et Clameurs au prix David ne jugeant pas [s]on livre inférieur à bien d'autres, mais ce qui n’implique nullement un degré de bonté. De toute façon, ça me fournira le luxe d'une brochure... (30 mai 1933) Il ne l'obtiendra pas, mais loin de se décourager, il entreprend la rédaction de contes et y travaille énormément (27 septembre 1933), ce qui se trouve confirmé dans une de ses lettres [toujours inédite] à Olivar Asselin: Dans ce temps-ci, je travaille comme un possédé des contes que je devrais avoir fini vers le 15 novembre. J'ai la tête en feu et les poches vides. (11 octobre 1933) Il s'agit sans doute du Déserteur et autres récits de la terre qu'il publiera l'année suivante, mais il y a aussi de fortes chances pour que l'un d'eux ne fut nul autre qu'Un homme et son péché qui ne devait être au départ qu'une nouvelle (et dont la rédaction ne lui aurait demandé qu'un mois). Il a déclaré dans ses Pamphlets n'avoir eu besoin ni de plan ni de composition arrêtée, écrivant à la hâte et comme pour se débarrasser du fardeau de la mémoire héréditaire dont parle Léon Daudet ${ }^{3}$. En effet, par sa lettre du 28 novembre, il en annonce la prochaine parution à DesRochers: $T u$ as sans doute entendu parler du roman qui doit paraître dans quelques jours et qui s'appellera pour toute l'Eternité, UN HOMME ET SON PÉCHÉ. C'est l'objet même de sa lettre:

Je n'ai pas besoin de te dire que cet ouvrage qui ne vaut rien n'a pas son comparable dans nos lettres, et c'est ce que j'écris à Bernard lui-même:' Pour une fois, et c'est ce que te dira l'éditeur si difficile, Alber.t Pelletier, le roman canayen paraît être écrit par un homme et peint des hommes. Et le péché. Ça se passe dans mon comté à l'époque de la colonisation. La vieille critique, si chère aux masturbateurs des deux sexes, va encore une fois rouspéter ou, ce qui est pis, ne dira pas un mot de mon livre, continuant de la sorte cette louable politique de la conspiration du silence. (28 novembre 1933)

C'est la raison pour laquelle Grignon demande à son ami de faire $d u$ train en maudit autour de ce livre. Il l'implore et le menace tour à tour:

3 Voir "Médecin, guéris-toi toi-même», les Pamphlets de Valdombre, $2^{e}$ année, $n^{\circ} 4$, mars 1938, p. 139. 
Je t'en conjure, fais-moi un article pour ton journal et pour ailleurs si tu peux. Tu ne dois pas ça à l'auteur, mais à l'Art Impersonnel. Autrement, tu n'es qu'un salaud, pas même digne de retourner à tes forêts. Penses-y sérieusement. Si nos propres amis ne font rien, les autres n'agiront pas, surtout si le livre a de la valeur. (28 novembre 1933)

DesRochers ne fera rien du tout. Avait-il jugé l'ouvrage trop terroiriste à son goût? Il se pourrait que tel fut le cas car Grignon, à l'occasion de la publication de Trente Arpents, qu'il était prêt à qualifier de chef-d'œuvre, en profitera pour faire l'apologie de la terre et de notre parlure et, de DesRochers, le chantre de la terre canadienne ${ }^{4}$. Mais ici, c'est beaucoup plus une ruade d'âne rancunier qu'un désir de louange. Il ne parle que d'À l'ombre de l'Orford, sachant bien que son ami lui préférait l'Offrande aux vierges folles, pour la seule raison que ce recueil s'éloignait précisément de la veine terroiriste de l'Orford. Grignon n'ignorait pas non plus que le poète suivait les conseils de Louis Dantin qui l'engageait à oublier l'Orford pour revenir à son style premier. Or, Grignon détestait souverainement Dantin. D'où son insistance à louer le canadianisme du poète de l'Orford:

Si DesRochers apporte un lyrisme vivifiant, s'il a la force de créer l'art, c'est parce qu'il reste intimement lié à la terre canadienne; c'est encore parce qu'il chante des mœurs, des coutumes qui sont de chez nous, ce qui n'exclut pas, au contraire, l'expression des passions humaines ou l'expression d'un art universel. ${ }^{5}$

La dernière lettre de Grignon date du 28 mars 1942 . Ce sera pour décliner une invitation du poète à donner une conférence à Sherbrooke. Grignon commence sa lettre par ces mots: Une lettre de toi me rappelle de bien chers souvenirs et me cause une grande surprise. Je crois ressusciter, qui laisserait entendre que ce serait DesRochers qui aurait mis fin à cette correspondance. La raison du refus de Grignon ne tient pas debout. Lui, qui adorait parler en public, ainsi qu'en témoignent les commentaires qu'il écrivait en marge de ses conférences, prétend que celles-ci l'épuisent et le laissent étourdi pendant des semaines. Il faut croire qu'il ne tenait pas à renouer avec le passé, cette époque chérie qui ne reviendrait plus, selon les derniers mots de sa lettre à DesRochers.

4 "Les Trente Arpents d'un Canayen ou le triomphe du régionalisme", les Pamphlets de Valdombre, $3^{\mathrm{e}}$ année, $n^{\circ} 3$, février 1939, p. 140-141. Ibid. 